\title{
Non-Abelian Bosonization in Two Dimensions
}

\author{
Edward Witten \\ Joseph Henry Laboratories, Princeton University, Princeton, NJ 08544, USA
}

\begin{abstract}
A non-abelian generalization of the usual formulas for bosonization of fermions in $1+1$ dimensions is presented. Any fermi theory in $1+1$ dimensions is equivalent to a local bose theory which manifestly possesses all the symmetries of the fermi theory.
\end{abstract}

One of the most startling aspects of mathematical physics in $1+1$ dimensions is the existence of a (non-local) transformation from local fermi fields to local bose fields. Thus, consider the theory of a massless Dirac fermion:

$$
\mathscr{L}_{D}=\bar{\psi} i \not \chi \psi \text {. }
$$

This theory is equivalent [1] to the theory of a free massless scalar field:

$$
\mathscr{L}_{S}=\frac{1}{2} \partial_{\mu} \phi \partial^{\mu} \phi
$$

The fermi field $\psi$ has a relatively complicated and non-local expression [2] in terms of $\phi$. However, fermion bilinears such as $\bar{\psi} \gamma_{\mu} \psi$ or $\bar{\psi} \psi$ take a simple form in the bose language. For example, the current $J_{\mu}=\bar{\psi} \gamma_{\mu} \psi$ becomes in terms of $\phi$

$$
J_{\mu}=\frac{1}{\sqrt{\pi}} \varepsilon_{\mu \nu} \partial^{v} \phi .
$$

Similarly the chiral densities $\mathcal{O}_{ \pm}=\bar{\psi}\left(1 \pm \gamma_{5}\right) \psi$ become

$$
\mathcal{O}_{ \pm}=M \exp \pm i \sqrt{4 \pi} \phi \text {, }
$$

where the value of the mass $M$ depends on the precise normal ordering prescription that is used to define the exponential in (4).

By means of formulas like (3) and (4), the equivalence between the free Dirac theory and the free scalar theory can be extended to interacting theories. A perturbation of the free Dirac Lagrangian can be translated, via (3) and (4), into an equivalent perturbation of the free scalar theory. This procedure is remarkably

\footnotetext{
* Supported in part by NSF Grant PHY-80-19754
} 
useful for elucidating the properties of $1+1$ dimensional theories. Many phenomena that are difficult to understand in the fermi language have simple, semiclassical explanations in the bose language. A major limitation of the usual bosonization procedure, however, is that in the case of fermi theories with nonabelian symmetries, these symmetries are not preserved by the bosonization. For instance, a theory with $N$ free Dirac fields has a $\mathrm{U}(N) \times \mathrm{U}(N)$ chiral symmetry [actually $\mathrm{O}(2 N) \times \mathrm{O}(2 N)$, as we will see later]. Upon bosonization, this becomes a theory with $N$ free scalar fields. The diagonal fermi currents can be bosonized conveniently, as in Eq. (3), but the off-diagonal currents are complicated and nonlocal in the bose theory. [Although the free scalar theory with $N$ fields has an $\mathrm{O}(N)$ symmetry, this $\mathrm{O}(N)$ does not correspond to any subgroup of the fermion symmetry group.] For this reason, it is rather difficult [3] to bosonize non-abelian theories by the usual procedure. It is also sometimes difficult to understand via bosonization the realization of non-abelian global symmetries.

In this paper, an alternative bosonization procedure will be described which generalizes the usual one and can be used to bosonize any theory in a local way, while manifestly preserving all of the original symmetries. Unfortunately, the resulting bose theories are somewhat complicated.

First, we rewrite Eq. (3) for the currents in a way susceptible of generalization.

We define an element $U$ of the $U(1)$ or $\mathrm{O}(2)$ group by $U=\exp i \sqrt{4 \pi} \phi$. Then (3) can be written

$$
J_{\mu}=-\frac{i}{2 \pi} \varepsilon_{\mu \nu} U^{-1} \partial^{v} U=-\frac{i}{2 \pi} \varepsilon_{\mu \nu}\left(\partial^{v} U\right) \cdot U^{-1} .
$$

We have emphasized in (5) that the ordering of factors does not matter, because the group U(1) is abelian. In generalizing (5) we will have to be careful about factor ordering.

It is convenient to rewrite (5) in light cone coordinates. Let $x^{ \pm}=\left(x^{0} \pm x^{1}\right) / \sqrt{2}$. In these coordinates the Lorentz invariant inner product is $A_{\mu} B^{\mu}=A^{+} B^{-}+A^{-} B^{+}$ $=A_{+} B_{-}+A_{-} B_{+}$; the components of a vector obey $A_{+}=A^{-}, A_{-}=A^{+}$. If we normalize the Levi-Civita symbol so that $\varepsilon_{01}=+1=-\varepsilon_{+-}$, then (3) and (5) become

$$
\begin{gathered}
J_{+}=-\frac{1}{\sqrt{\pi}} \partial_{+} \phi=\frac{i}{2 \pi} U^{-1} \partial_{+} U, \\
J_{-}=+\frac{1}{\sqrt{\pi}} \partial_{-} \phi=-\frac{i}{2 \pi}\left(\partial_{-} U\right) U^{-1} .
\end{gathered}
$$

Of course, the ordering of factors in (6) is still arbitrary.

For the massless Dirac particle, the vector and axial vector currents $\bar{\psi} \gamma^{\mu} \psi$ and $\bar{\psi} \gamma^{\mu} \gamma_{5} \psi$ are both conserved. ${ }^{1}$ But in $1+1$ dimensions $\bar{\psi} \gamma^{\mu} \gamma_{5} \psi=\varepsilon^{\mu \nu} \bar{\psi} \gamma_{v} \psi$. So the current conservation equations are $0=\partial_{\mu} J^{\mu}=\varepsilon^{\mu v} \partial_{\mu} J_{v}$. In light cone coordinates this means $0=\partial_{-} J_{+}=\partial_{+} J_{-}$. The bosonization formula (6) is compatible with that strong condition because the free massless $\phi$ field obeys $0=\nabla^{2} \phi=2 \partial_{+} \partial_{-} \phi$.

1 As usual, we define $\left\{\gamma_{\mu}, \gamma_{\nu}\right\}=2 \eta_{\mu \nu}, \gamma_{5}=\gamma^{0} \gamma^{1}$ (so $\gamma_{5}^{2}=+1$ ), and $\bar{\psi}=\psi^{*} \gamma^{0}$. A convenient basis is $\gamma^{0}$ $=\left(\begin{array}{ll}0 & 1 \\ 1 & 0\end{array}\right), \gamma^{1}=\left(\begin{array}{rr}0 & -1 \\ 1 & 0\end{array}\right), \gamma_{5}=\left(\begin{array}{rr}1 & 0 \\ 0 & -1\end{array}\right)$. We define light cone components $\psi_{ \pm}$of $\psi$ by requiring $\gamma_{5} \psi_{-}$ $=\psi_{-}, \gamma_{5} \psi_{+}=-\psi_{+} \cdot$ (The sign convention may seem odd but is useful.) Thus $\psi=\left(\begin{array}{c}\psi_{+} \\ \psi_{-}\end{array}\right) \cdot \psi_{+}$and $\psi_{-}$are
left movers and right movers, respectively, as one may see from Eq. (8) later 
We wish to generalize this to fermion theories with non-abelian symmetries. As we wish to be general, we will consider a theory with $N$ Majorana fermions $\psi^{i}$, $i=1 \ldots N$. [If one prefers, one can choose $N$ even and consider this to be a theory of $N / 2$ Dirac fields. If so, in much of the subsequent discussion one can consider the chiral group $\mathrm{U}(N / 2) \times \mathrm{U}(N / 2)$ instead of $\mathrm{O}(N) \times \mathrm{O}(N)$.] The conventional Lagrangian for free Majorana fields is

$$
\mathscr{L}=\int d^{2} x \frac{1}{2} \bar{\psi}_{k} i \not \partial \psi^{k} .
$$

The conserved vector currents are $V_{\mu}^{a}=\bar{\psi} \gamma_{\mu} T^{a} \psi, T^{a}$ being any generator of $\mathrm{O}(N)$. The axial currents are $A_{\mu}^{a}=\varepsilon_{\mu \nu} V^{v a}=\bar{\psi} \gamma_{\mu} \gamma_{5} T^{a} \psi$. These currents generate chiral $\mathrm{O}(N) \times \mathrm{O}(N)$.

Since $\bar{\psi} \gamma^{\mu} \partial_{\mu} \psi=\psi^{T}\left(\partial_{0}+\gamma^{0} \gamma^{1} \partial_{1}\right) \psi$, the free Lagrangian, in terms of the light cone components of $\psi$, is

$$
\mathscr{L}=\frac{1}{2} i \int d^{2} x\left[\psi_{-}^{k}\left(\frac{\partial}{\partial t}+\frac{\partial}{\partial x}\right) \psi_{-}^{k}+\psi_{+}^{k}\left(\frac{\partial}{\partial t}-\frac{\partial}{\partial x}\right) \psi_{+}^{k}\right] .
$$

Instead of vector and axial vector currents, it is more useful to work with chiral components. We define $J_{+}^{i j}(x, t)=-i \psi_{+}^{i} \psi_{+}^{j}(x, t)$ and $J_{-}^{i j}(x, t)=-i \psi_{-}^{i} \psi_{-}^{j}(x, t)$. Note that $J_{ \pm}^{i j}$ are hermitian and that by fermi statistics they obey $J_{+}^{i j}=-J_{+}^{j i}, J_{-}^{i j}=-J_{-}^{j i}$. $J_{+}^{i j}$ and $J_{-}^{i j}$ generate chiral $\mathrm{O}(N)_{R}$ and $\mathrm{O}(N)_{L}$, respectively. $\left[\mathrm{By} \mathrm{O}(N)_{R}\right.$ and $\mathrm{O}(N)_{L}$ we mean $\mathrm{O}(N)$ transformations for right-moving and left-moving fermions.] The conservation laws for $J_{ \pm}$are very simple

$$
\partial_{-} J_{+}^{i j}=\partial_{+} J_{-}^{i j}=0 \text {. }
$$

Thus, $J_{+}$is a function only of $x^{+}$, and $J_{-}$is a function only of $x^{-}$.

We wish to find an ansatz writing $J_{+}$and $J_{-}$in terms of suitable bose fields. In the usual bosonization procedure, one considers a current $\bar{\psi} \gamma_{\mu} \psi$ that generates an abelian or $\mathrm{U}(1)$ symmetry; it is written [Eq. (5)] in terms of a field that takes values in the U(1) group. Now we are dealing with currents $J_{-}^{i j}$ and $J_{+}^{i j}$ that generate $\mathrm{O}(N)_{L} \times \mathrm{O}(N)_{R}$, and it is natural to try to express these currents in terms of a suitable field $g$ that takes values in the $\mathrm{O}(N)$ group. $\mathrm{O}(N)_{L} \times \mathrm{O}(N)_{R}$ will act on $g$ by $g \rightarrow A g B^{-1}, A, B \in \mathrm{O}(N)$.

What is a suitable expression for the currents in terms of $g$ ? One is tempted to $\operatorname{try} J_{+} \sim g^{-1} \partial_{+} g, J_{-} \sim g^{-1} \partial_{-} g$. However, this is incompatible with (9) because in a non-abelian group the equations $0=\partial_{-}\left(g^{-1} \partial_{+} g\right)$ and $0=\partial_{+}\left(g^{-1} \partial_{-} g\right)$ are inconsistent. Instead, we generalize the factor ordering of Eq. (6) and write

$$
J_{+}=\frac{i}{2 \pi} g^{-1} \partial_{+} g, \quad J_{-}=-\frac{i}{2 \pi}\left(\partial_{-} g\right) g^{-1} .
$$

[The ij indices are suppressed, it being understood that $J_{+}$and $J_{-}$are elements of the $\mathrm{O}(N)$ Lie algebra.] Notice that the equations $0=\partial_{-}\left(g^{-1} \partial_{+} g\right)$ and $0=\partial_{+}\left(\left(\partial_{-} g\right) g^{-1}\right)$ are compatible and in fact equivalent.

What Lagrangian will govern $g$ ? The obvious guess is

$$
\mathscr{L}=\frac{1}{4 \lambda^{2}} \operatorname{Tr} \partial_{\mu} g \partial_{\mu} g^{-1} .
$$

This is the unique renormalizable and manifestly chirally invariant Lagrangian for $g$. However, for many reasons, (11) is wrong. 


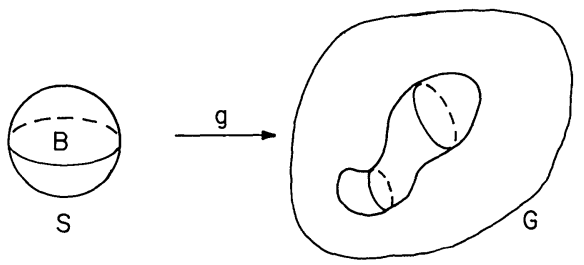

Fig. 1. A mapping $g$ from a two sphere $S$ (representing space-time) into a group manifold $G$. Since $\pi_{2}(G)=0$, any mapping of the surface $S$ into $G$ can be extended to a mapping into $G$ of the solid sphere $B$ ( $S$ and its interior)

First of all, (11) describes an asymptotically free theory with interactions that become strong in the infrared. It is certainly not equivalent to the conformally invariant free massless fermi field theory. Second, (11) leads to the equation of motion $0=\partial_{\mu}\left(g^{-1} \partial_{\mu} g\right)$ rather than the desired $0=\partial_{-}\left(g^{-1} \partial_{+} g\right)=\partial_{+}\left(\left(\partial_{-} g\right) g^{-1}\right)$. Third, by analogy with similar considerations in QCD current algebra, [8] it may be shown that (11) has more discrete symmetries than the free, massless fermi theory.

Although (11) is the only renormalizable interaction for the non-linear sigma model that is manifestly chirally invariant, there is another one that is chirally invariant but not manifestly so. This is the two-dimensional analogue of the WessZumino term [4], which has figured in various recent discussions of two dimensional models [5-7].

The two dimensional Wess-Zumino term can be constructed by analogy [8] with a similar treatment in four dimensions. Working in Euclidean space, we imagine space time to be a large two sphere $S^{2}$. Since $\pi_{2}(\mathrm{O}(N))=0$, a mapping $g$ from $S$ into the $\mathrm{O}(N)$ manifold can be extended to a mapping $\bar{g}$ of a solid ball $B$ whose boundary is $S$ into $\mathrm{O}(N)$ (Fig. 1). If $y_{1}, y_{2}$, and $y_{3}$ are coordinates for $B$, the Wess-Zumino functional is

$$
\Gamma=\frac{1}{24 \pi} \int_{B} d^{3} y \varepsilon^{i j k} \operatorname{Tr} \bar{g}^{-1} \frac{\partial \bar{g}}{\partial y^{i}} \bar{g}^{-1} \frac{\partial \bar{g}}{\partial y^{j}} \bar{g}^{-1} \frac{\partial \bar{g}}{\partial y^{k}} .
$$

As in four dimensions, the Wess-Zumino functional has a very essential property $[8,9]$ : it is well-defined only modulo a constant. Equation (12) has been normalized so that if $g$ is a matrix in the fundamental representation of $\mathrm{O}(N),(12)$ is well-defined modulo $\Gamma \rightarrow \Gamma+2 \pi$. The ambiguity in $\Gamma$ arises because of the existence of topologically inequivalent ways to extend $g$ into a mapping from $B$ into $\mathrm{O}(N)$; the topologically distinct possibilities are classified by $\pi_{3}(\mathrm{O}(N)) \simeq Z$.

In what sense is $\Gamma$ an ordinary Lagrangian - an integral over space-time? This question is answered in the appendix, where it is shown that (locally in field space) $\Gamma$ can be written as the integral over space-time of an ordinary but not manifestly chirally invariant Lagrangian which under a chiral transformation changes by a total divergence.

2 In Minkowski space, we instead consider space to be compact. We then consider finite time transition amplitudes between specified initial and final states of the $g$ field. This "ties down" the fields at the boundary of space-time and leads to a similar quantization argument for $\Gamma$ 
Making use of $\Gamma$, we can consider a more general action for the field $g$ :

$$
I=\frac{1}{4 \lambda^{2}} \int d^{2} x \operatorname{Tr} \partial_{\mu} g \partial^{\mu} g^{-1}+n \Gamma .
$$

Here $n$ must be an integer [19], since $\Gamma$ is well-defined only modulo $2 \pi$. The theory (13) is renormalizable, since the new coupling constant is a dimensionless integer. Perhaps it should be stressed that (13) is not invariant under naive parity $x \rightarrow-x$, but is invariant under $x \rightarrow-x, g \rightarrow g^{-1}$.

We wish to ask whether for some values of $\lambda$ and $n$ this theory might be equivalent to the free massless fermi theory.

The first step is to calculate the equations of motion from (13). As has been discussed previously $[6,8]$, the variation of $\Gamma$ is a simple, local functional. We find from (13) that the change of $I$ under $g \rightarrow g+\delta g$ is

$$
\delta I=\frac{1}{2 \lambda^{2}} \int d^{2} \operatorname{Tr} g^{-1} \delta g \partial_{\mu}\left(g^{-1} \partial_{\mu} g\right)-\frac{n}{8 \pi} \int d^{2} x \operatorname{Tr} g^{-1} \delta g \varepsilon^{\mu \nu} \partial_{\mu}\left(g^{-1} \partial_{\nu} g\right) .
$$

The variational equations are therefore

$$
\begin{aligned}
0 & =\frac{1}{2 \lambda^{2}} \partial_{\mu}\left(g^{-1} \partial_{\mu} g\right)-\frac{n}{8 \pi} \varepsilon^{\mu \nu} \partial_{\mu}\left(g^{-1} \partial_{\nu} g\right) \\
& =\left(\frac{1}{2 \lambda^{2}}+\frac{n}{8 \pi}\right) \partial_{-}\left(g^{-1} \partial_{+} g\right)+\left(\frac{1}{2 \lambda^{2}}-\frac{n}{8 \pi}\right) \partial_{+}\left(g^{-1} \partial_{-} g\right) .
\end{aligned}
$$

We see therefore that if $\lambda^{2}=\frac{4 \pi}{n}$ the equation is as desired, $0=\partial_{-}\left(g^{-1} \partial_{+} g\right)$. Of course, $\lambda^{2}$ must be positive for stability, so this is only possible for $n>0$. For $n<0$ the parity conjugate equation $0=\partial_{+}\left(g^{-1} \partial_{-} g\right)$ arises at $\lambda^{2}=-\frac{4 \pi}{n}$.

At $\lambda^{2}=\frac{4 \pi}{n}$ the equations of motion of the theory can easily be solved in closed form. The general solution of $0=\partial_{-}\left(g^{-1} \partial_{+} g\right)$ is

$$
g\left(x^{+}, x^{-}\right)=A\left(x^{-}\right) B\left(x^{+}\right),
$$

where $A\left(x^{-}\right)$and $B\left(x^{+}\right)$are arbitrary $\mathrm{O}(N)$ valued functions of one coordinate. [At $\lambda^{2}=-4 \pi / n$ the factorization is instead $g\left(x^{+}, x^{-}\right)=B\left(x^{+}\right) A\left(x^{-}\right)$.] Equation (16) means that left-moving and right-moving waves pass through each other without any interference. This property is strongly reminiscent of the fermion free field theory, in which the left- and right-moving waves are the $\gamma_{5}$ eigenstates. Combining this analogy with the fact that at $\lambda^{2}=4 \pi / n$ the equation of motion for $g$ reproduces the behavior of the fermion currents, we are led to conjecture that at $\lambda^{2}=4 \pi / n$ and some value of $n$ the non-linear sigma model is equivalent to the fermion free field theory.

What are the renormalization group properties of the theory with action (13)? Being an integer, $n$ must not be subject to renormalization. This can be established in the background field method; in that method the counter-terms are local and manifestly chinally invariant functionals of the background field, so there is no 


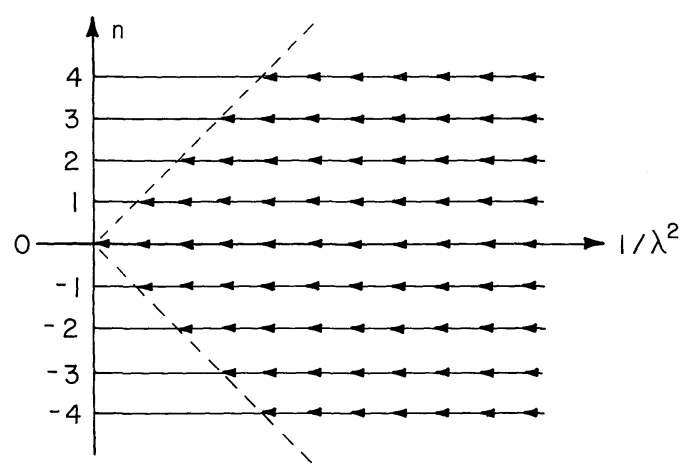

Fig. 2. Renormalization group flows. Plotted is the behavior of $1 / \lambda^{2}$ in coming from high-energy to lowenergy. Weak coupling is at the right, strong coupling is at the left. Assuming there are no non-trivial fixed points apart from the one found in the text, the $n=0$ theory flows to strong coupling at long distances, while for $n \neq 0$ the theory flows to $\lambda^{2}=|4 \pi / n|$. The behaviour of the non-asymptotically free theory with bare coupling bigger than $|4 \pi / n|$ is not considered

counterterm proportional to $\Gamma^{3}$. We will illustrate this shortly at the one loop level.

The theory therefore requires only renormalization of $\lambda$. However, the renormalization of $\lambda$ depends on both $\lambda$ and $n$. For any $n$, the theory is asymptotically free, just as at $n=0$. This is so because for $\lambda$ so small that $\frac{1}{\lambda^{2}} \gg n,(13)$ is dominated by the first term, and the renormalization group calculation coincides with the standard calculation at $n=0$. However, as $\lambda$ becomes large, the effects of the Wess-Zumino term can become important. We will argue that the beta function always has a zero at $\lambda^{2}= \pm \frac{4 \pi}{n}$. We will first illustrate this point with a one loop calculation; then we will establish the point by showing that the theory at $\lambda^{2}= \pm 4 \pi / n$ is equivalent to a known exactly soluble, conformally invariant theory. The existence of a non-trivial zero of the beta function at $\lambda^{2}=\frac{4 \pi}{n}$ means that the physical content of the weakly coupled theory with $n \neq 0$ is dramatically different from what it is for $n=0$. Instead of flowing in the infrared to strong coupling, the coupling constant flows (Fig. 2) to $\sqrt{4 \pi / n}$ (or perhaps to another zero of the beta function closer to the origin).

Let us now calculate the one loop beta function of the theory. We will use the background field and expand around an arbitrary solution $g_{0}$ of the classical field equations. We write $g=g_{0} \exp i \lambda T^{a} \pi^{a}$, where the $T^{a}$ (normalized so $\operatorname{Tr} T^{a} T^{b}=2 \delta^{a b}$ ) are the generators of $\mathrm{O}(N)$ and $\pi^{a}$ are the small fluctuation fields. The action becomes

$$
\begin{aligned}
I= & \int d^{2} x\left[\frac{1}{4 \lambda^{2}} \operatorname{Tr} \partial_{\mu} g_{0} \partial_{\mu} g_{0}^{-1}+\frac{1}{2} \sum_{a}\left(\partial_{\mu} \pi^{a}\right)^{2}+\frac{\eta^{\mu \nu}}{4} \operatorname{Tr} g_{0}^{-1} \partial_{\mu} g_{0}\left[T \cdot \pi, \partial_{\nu} T \cdot \pi\right]\right. \\
& \left.-\frac{1}{4} \frac{\lambda^{2} n}{4 \pi} \varepsilon^{\mu \nu} \operatorname{Tr} g_{0}^{-1} \partial_{\mu} g_{0}\left[T \cdot \pi, \partial_{\nu} T \cdot \pi\right]\right]
\end{aligned}
$$

3 Similar reasoning has been given in discussion of the $\theta$ angle in four dimensions by Novikov, Shifman, Vainshtain, and Zakharov (private communication) 


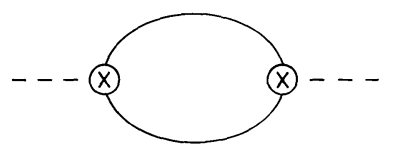

Fig. 3. The one loop renormalization calculation in the nonlinear sigma model. The dotted line is the background field; the solid line represents quantum fluctuations. The divergence arises only if the two vertices contain both $\eta^{\mu v}$ or both $\varepsilon^{\mu v}$

up to terms cubic or higher order in $\pi$. By power counting, a one loop divergence will be quadratic in $g_{0}^{-1} \partial_{\mu} g_{0}$. The only possible quadratic term is $\operatorname{Tr}\left(g_{0}^{-1} \partial_{\mu} g_{0}\right)^{2}$ $=-\operatorname{Tr} \partial_{\mu} g_{0} \partial_{\mu} g_{0}^{-1}$, since $\varepsilon^{\mu \nu} \operatorname{Tr}\left(g_{0}^{-1} \partial_{\mu} g_{0}\right)\left(g_{0}^{-1} \partial_{\nu} g_{0}\right)=0$. This shows at the one loop level that only renormalization of $\lambda$ is necessary - as was asserted earlier.

Since the sought for counterterm $\operatorname{Tr} \partial_{\mu} g_{0} \partial_{\mu} g_{0}^{-1}$ is even under naive parity $x \rightarrow-x, g \rightarrow g$, the divergent one loop diagrams (Fig. 3) have two vertices both proportional to $\eta^{\mu v}$ or both proportional to $\varepsilon^{\mu v}$. The $\eta^{\mu v}$ vertex is the usual one that give asymptotic freedom [10]. The $\varepsilon^{\mu v}$ vertex is known from an old calculation in a different model [11] to give a positive contribution to the beta function. Actually even without evaluating the diagrams it is easy to see that they cancel if $\lambda^{2}= \pm \frac{4 \pi}{n}$. Apart from a factor of $\left(\frac{\lambda^{2} n}{4 \pi}\right)^{2}$, they differ in that one diagram has a factor of $\eta_{\mu}^{\alpha} \eta_{\alpha \nu}=\eta_{\mu \nu}$ while the other has $\varepsilon_{\mu}^{\alpha} \varepsilon_{v \alpha}=-\eta_{\mu \nu}$. Actual evaluation of the diagrams of Fig. 3 is not difficult. The divergent term in the effective action is

$$
\int \frac{i(N-2)}{16 \pi} \operatorname{Tr} \partial_{\mu} g_{0} \partial_{\mu} g_{0}^{-1} \ln \left(\frac{\Lambda^{2}}{\mu^{2}}\right) d^{2} x,
$$

where $\Lambda$ is a momentum space cut-off and $\mu$ is a renormalization mass. From this we read off the one loop beta function

$$
\beta(\lambda, n)=-\frac{\lambda^{2}(N-2)}{4 \pi}\left[1-\left(\frac{\lambda^{2} n}{4 \pi}\right)^{2}\right]
$$

which, as claimed, vanishes for $\lambda^{2}=\left|\frac{4 \pi}{n}\right|$.

If $n$ is very large, say $n=10^{10}$, this perturbative calculation reliably shows the existence of a zero of the beta function, since the computed zero is at a very small coupling for which higher order terms are negligible. Of course, this reasoning does not show that the zero of the beta function is precisely at $|4 \pi / n|$; and for $n$ of order one the lowest order calculation does not reliably show even the existence of a zero. To show that the beta function vanishes for $\lambda^{2}=|4 \pi / n|$ and that the theory at the zero is exactly soluble requires more information.

Let us return to the fermion currents, $J_{+}^{i j}=-i \psi_{+}^{i} \psi_{+}^{j}, J_{-}^{i j}=-i \psi_{-}^{i} \psi_{-}^{j}$, and to our hypothesis that these currents can be equated with suitable expressions constructed from $g$. What commutation relations do the fermion currents obey? The canonical anticommutation relations for the fermi fields are $\left\{\psi_{+}^{i}(x), \psi_{+}^{j}(y)\right\}$ $=\left\{\psi_{-}^{i}(x), \psi_{-}^{j}(y)\right\}=\delta^{i j} \delta(x-y),\left\{\psi_{-}^{i}(x), \psi_{-}^{j}(y)\right\}=0$. Using these equations one can readily work out the canonical commutation rules for $J_{ \pm}$. These canonical 


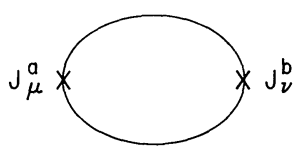

Fig. 4. The one loop diagram that yields the Schwinger anomaly in $1+1$ dimensions

relations, however, are not valid quantum mechanically. The proper quantum mechanical formulas contain a $c$-number anomaly term, the Schwinger term. It arises [12] from diagram (4) ${ }^{4}$. The quantum mechanical commutation relations can be compactly written

$$
\begin{aligned}
{\left[\operatorname{Tr} A J_{-}(x), \operatorname{Tr} B J_{-}(y)\right] } & =2 i \delta(x-y) \operatorname{Tr}[A, B] J_{-}(x)+\frac{i}{\pi} \delta^{\prime}(x-y) \operatorname{Tr} A B, \\
{\left[\operatorname{Tr} A J_{+}(x), \operatorname{Tr} B J_{+}(y)\right] } & =2 i \delta(x-y) \operatorname{Tr}[A, B] J_{+}(x)-\frac{i}{\pi} \delta^{\prime}(x-y) \operatorname{Tr} A B, \\
{\left[J_{-}^{i j}, J_{+}^{k l}\right] } & =0,
\end{aligned}
$$

where $A$ and $B$ are arbitrary antisymmetric matrices [generators of $\mathrm{O}(N)]$. The terms proportional to $\delta^{\prime}(x-y)$ originate from the anomaly.

Consider the following generalization of the first line of Eq. (20):

$$
\left[\operatorname{Tr} A J_{-}(x), \operatorname{Tr} B J_{-}(y)\right]=2 i \delta(x-y) \operatorname{Tr}[A, B] J_{-}(x)+k \frac{i}{\pi} \delta^{\prime}(x-y) \operatorname{Tr} A B .
$$

Here we allow the coefficient of the anomaly to be rescaled by an arbitrary constant $k$. This algebra is known in the mathematical literature as the KacMoody algebra with a central extension, the central extension being $k \neq 0$ [13]. In the mathematical literature it is shown that this algebra has well-behaved unitary representations if and only if $k$ is an integer. Actually, in quantum field theory one can easily find a system in which the anomaly has an arbitrary integer strength $k$. Consider a theory with $k$ "flavors" and $N$ "colors" of fermions $\psi^{i a}, a=1 \ldots k$, $i=1 \ldots N$, and define $J_{-}^{i j}=-i \sum_{a=1}^{k} \psi_{-}^{i a} \psi_{-}^{j k}$. The anomaly is then $k$ times as large, coming from a sum over the flavor index in Fig. 4. [This gives an arbitrary positive integer $k$ in (21); if a negative integer is desired, one may consider $J_{+}$instead.] The fact that the Kac-Moody representation theory is well behaved only for integral $k$ is another aspect of the a priori quantization of anomalies, a phenomenon that can also be seen from instanton physics [14] or from the multivaluedness of the WessZumino term [8].

Our one flavor theory obeys (20) with $k= \pm 1$. The following very important facts are known about the Kac-Moody algebra. The unitary irreducible representation for $k= \pm 1$ is essentially unique. For $k>1$, there are a finite number of irreducible representations, obtained by taking tensor products of the $k=1$ representation with different symmetry or antisymmetry conditions. To prove the equivalence of a boson theory to the one flavor fermion theory it is sufficient to show that the boson theory gives currents that obey a Kac-Moody algebra with $k= \pm 1$.

4 The evaluation of the anomaly is standard. One derivation of this formula is described in detail by Coleman et al. [12, Eqs. (3.4), (4.19), and (4.28)]. They use, however, a notation based on Dirac fermions 


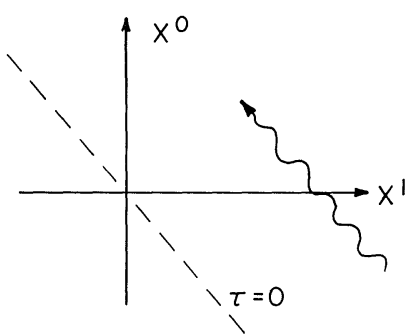

Fig. 5. This diagram is meant to illustrate the limitations of a canonical formalism based on light cone coordinates in $1+1$ dimensions. The dotted line is an "initial value surface", $\tau=0\left(\tau=\left(x^{0}+x^{1}\right) / \sqrt{2}\right)$. The wave line is a massless particle traveling to the left at the speed of light. Its world path never meets $\tau=0$, so its existence cannot be predicted from initial data at $\tau=0$

We are thus led to try to calculate the canonical commutation relations of the currents $g^{-1} \partial_{+} g$ and $\left(\partial_{-} g\right) g^{-1}$. Actually, we will calculate the purely classical Poisson bracket (PB). This calculation may appear formidable because of the complexity of the Wess-Zumino term but in fact at the critical coupling $\lambda^{2}=\left|\frac{4 \pi}{n}\right|$ it can be carried out more or less simply.

First of all, since $g^{-1} \partial_{+} g$ is only a function of $x^{+}$and $\left(\partial_{-} g\right) g^{-1}$ is only a function of $x^{-}$, their Poisson bracket $\left[\left(g^{-1} \partial_{+} g\right)_{i j},\left(\left(\partial_{-} g\right) g^{-1}\right)_{k l}\right]_{\mathrm{PB}}$ vanishes. It is enough to calculate the Poisson bracket of $\left(\partial_{-} g\right) g^{-1}$ with itself; the Poisson bracket of $g^{-1} \partial_{+} g$ can be deduced from the symmetry under $x \leftrightarrow-x, g \leftrightarrow g^{-1}$.

We will carry out the canonical analysis in a "light-cone frame." This means that we will regard $\sigma=x^{-}=\frac{x^{0}-x^{1}}{\sqrt{2}}$ as "space" while regarding $\tau=x^{+}=\frac{x^{0}+x^{1}}{\sqrt{2}}$ as "time." Actually, in $1+1$ dimensions the light cone framework has a drawback. A left-moving massless degree of freedom may be unpredictable on the basis of initial data at $\tau=0$ (Fig. 5). For this reason, the light cone treatment fails to give the Poisson bracket of operators like $g^{-1} \partial_{+} g$ that contain $\tau$ derivatives. (Their Poisson brackets can be obtained from an opposite light cone treatment in which $\tau$ is regarded as space and $\sigma$ as time.) But the light cone framework yields straightforwardly the Poisson brackets of operators like $\left(\partial_{-} g\right) \cdot g^{-1}$ that do not contain $\tau$ derivatives.

With $\lambda^{2}=4 \pi / n$, the action, in light cone coordinates, is

$$
I=\frac{n}{16 \pi} \int d \sigma d \tau \operatorname{Tr} \partial_{\tau} g \partial_{\sigma} g^{-1}+n \Gamma .
$$

$\Gamma$ is rather complicated but it has one simple property: it is first order in time derivatives. Therefore, the whole action (22) has this property.

To introduce a canonical formalism it is necessary to formulate a theory with an action that is first order in time derivatives. Usually this requires introducing momenta that are independent of the coordinates, passing for instance from $\frac{1}{2} \dot{q}^{2}-V$ to $p \dot{q}-\frac{1}{2} p^{2}-V$. The case at hand is an exception. Equation (22) is already in Hamiltonian form; that is, it is already of first order in time derivatives. 
In one other way, (22) differs from usual experience. In the light cone nonlinear sigma model it is not convenient to split the dynamical variables into coordinates and momenta. Let us discuss, therefore, how Poisson brackets may in general be computed without making an explicit choice of $p$ 's and $q$ 's.

Consider a theory with dynamical variables $\phi^{i}$ and an arbitrary action that is first order in time derivatives:

$$
I=\int d t A_{i}(\phi) \frac{d \phi^{i}}{d t}
$$

(The action may also contain terms independent of time derivatives; such terms are ignored in computing Poisson brackets.) We calculate the change in $I$ under an arbitrary infinitessimal variation $\phi^{i} \rightarrow \phi^{i}+\delta \phi^{i}$ :

$$
\begin{aligned}
\delta I & =\int d t\left(\frac{\partial A_{i}}{\partial \phi^{j}} \delta \phi^{j} \frac{d \phi^{i}}{d t}+A_{i} \frac{d}{d t} \delta \phi^{i}\right) \\
& =\int d t\left(\frac{\partial A_{i}}{\partial \phi^{j}} \delta \phi^{j} \frac{d \phi^{i}}{d t}-\frac{d A_{i}}{d t} \delta \phi^{i}\right) \\
& =\int d t\left(\frac{\partial}{\partial \phi^{i}} A_{j}-\frac{\partial}{\partial \phi^{j}} A_{i}\right) \delta \phi^{i} \frac{d \phi^{j}}{d t} .
\end{aligned}
$$

Define a matrix $F_{i j}=\partial_{i} A_{j}-\partial_{j} A_{i}$ as the coefficient of $\delta \phi^{i} \frac{d \phi^{j}}{d t}$. Notice that $F_{i j}$ is always antisymmetric. Let $F^{j k}$ be the universe matrix of $F_{i j}\left(\right.$ so $\left.F^{j k} F_{k i}=\delta_{i}^{j}\right)$. ${ }^{5}$ Then the Poisson bracket of any two functions on phase space $X$ and $Y$ is defined by

$$
[X, Y]_{\mathrm{PB}}=\sum_{i, j} F^{i j} \frac{\partial X}{\partial \phi^{i}} \frac{\partial Y}{\partial \phi^{j}} .
$$

In the simple case in which the $\phi^{i}$ are decomposed into coordinates and momenta $q^{i}$ and $p^{i}$, and in which the part of the action containing time derivatives is $\int d t \sum_{i} p^{i} \frac{d q^{i}}{d t},(25)$ agrees with the usual definition of Poisson brackets.

In this calculation it is unnecessary to choose an explicit set of coordinates $\phi^{i}$ for the classical phase space. (Such a choice would be very awkward in the nonlinear sigma model becuase of the nonlinearity of the phase space.) It is enough to have a basis of tangent vectors to the phase space (analogous to the tetrad in general relativity). The matrices $F_{i j}$ and $F^{j k}$ may be constructed relative to any such basis. In the non-linear sigma model a very convenient basis of tangents to the phase space are the matrices $g^{-1} \delta g(\sigma)$. The matrix $F$ must act both on the Lie algebra index of $g^{-1} \delta g(\sigma)$ and on $\sigma$.

In this basis, it is very easy to calculate the matrix $F$ in the non-linear sigma model. From (14), with $\lambda^{2}=4 \pi / n$, the variation of the action is

$$
\delta I=\frac{n}{4 \pi} \int d \sigma d \tau \operatorname{Tr} g^{-1} \delta g \frac{d}{d \sigma} g^{-1} \frac{d g}{d t} .
$$

5 If this inverse does not exist, one must introduce "constraints.") This does not occur in the case at hand 
From (26) we see that $F$ is $1 \otimes \frac{n}{4 \pi} \frac{d}{d \sigma}$, where " 1 " acts on the Lie algebra index and $\frac{n}{4 \pi} \frac{d}{d \sigma}$ acts on the spatial coordinate. The inverse matrix is, of course $1 \otimes \frac{4 \pi}{n}\left(\frac{d}{d \sigma}\right)^{-1}$

We now wish to apply definition (25) of the Poisson bracket with $X=\operatorname{Tr} A \frac{d g}{d \sigma} g^{-1}(\sigma), \quad Y=\operatorname{Tr} B \frac{d g}{d \sigma} g^{-1}\left(\sigma^{\prime}\right)$. Note that (25) can be understood as follows. First calculate $\delta X \delta Y=\frac{\partial X}{\partial \phi^{i}} \frac{\partial Y}{\partial \phi^{j}} \delta \phi^{i} \delta \phi^{j}$; then replace $\delta \phi^{i} \delta \phi^{j}$ by $F^{i j}$. So we calculate $\delta X$ :

$$
\begin{aligned}
\delta X & =\operatorname{Tr} A\left(\frac{d}{d \sigma} \delta g\right) g^{-1}(\sigma)+\operatorname{Tr} A \frac{d g}{d \sigma} \delta g^{-1} \\
& =\operatorname{Tr} A\left(\frac{d}{d \sigma} \delta g\right) g^{-1}-\operatorname{Tr} A \frac{d g}{d \sigma} g^{-1} \delta g g^{-1} \\
& =\operatorname{Tr} g^{-1}(\sigma) A g(\sigma) \frac{d}{d \sigma}\left(g^{-1} \delta g(\sigma)\right)
\end{aligned}
$$

$\delta Y$ is evaluated similarly, so

$$
\delta X \delta Y=\operatorname{Tr} g^{-1}(\sigma) A g(\sigma) \frac{d}{d \sigma}\left(g^{-1} \delta g(\sigma)\right) \cdot \operatorname{Tr} g^{-1}\left(\sigma^{\prime}\right) B g\left(\sigma^{\prime}\right) \frac{d}{d \sigma^{\prime}}\left(g^{-1} \delta g\left(\sigma^{\prime}\right)\right) .
$$

After evaluating $\delta X \delta Y=\frac{\partial X}{\partial \phi^{i}} \frac{\partial Y}{\partial \phi^{j}} \delta \phi^{i} \delta \phi^{j}$, the next step is to replace $\delta \phi^{i} \delta \phi^{j}$ with $F^{i j}$. In our problem the role of $\delta \phi^{i}$ and $\delta \phi^{j}$ is played by $\left(g^{-1} \delta g(\sigma)\right)^{a}$ and $\left(g^{-1} \delta g\left(\sigma^{\prime}\right)\right)^{b}$ (here we explicitly exhibit - temporarily - the Lie algebra indices $a$ and $b$ carried by these matrices). In view of our previous determination of $F_{i j}$ and $F^{i j}$, we are to replace $\left(g^{-1} \delta g(\sigma)\right)^{a}\left(g^{-1} \delta g(\sigma)\right)^{b}$ by $\delta^{a b} \frac{4 \pi}{n} \theta\left(\sigma, \sigma^{\prime}\right)$ where $\theta\left(\sigma, \sigma^{\prime}\right)$ is an inverse of $\frac{d}{d \sigma}$. Hence $\frac{d}{d \sigma}\left(g^{-1} \delta g(\sigma)\right)^{a} \cdot \frac{d}{d \sigma^{\prime}}\left(g^{-1} \delta g\left(\sigma^{\prime}\right)\right)^{b}$ is replaced by $\delta^{a b} \frac{4 \pi}{n} \frac{d}{d \sigma}$ $\cdot \frac{d}{d \sigma^{\prime}} \theta\left(\sigma-\sigma^{\prime}\right)=-\delta^{a b} \frac{4 \pi}{n} \delta^{\prime}\left(\sigma-\sigma^{\prime}\right)$. For the Poisson bracket of $X$ and $Y$ we get therefore

$$
\begin{aligned}
{[X, Y]_{\mathrm{PB}} } & =-\frac{4 \pi}{n} \delta^{\prime}\left(\sigma-\sigma^{\prime}\right) \operatorname{Tr} g^{-1}(\sigma) A g(\sigma) g^{-1}\left(\sigma^{\prime}\right) B g\left(\sigma^{\prime}\right) \\
& =-\frac{4 \pi}{n} \delta\left(\sigma-\sigma^{\prime}\right) \operatorname{Tr}[A, B] \frac{d g}{d \sigma} g^{-1}-\frac{4 \pi}{n} \delta^{\prime}\left(\sigma-\sigma^{\prime}\right) \operatorname{Tr} A B
\end{aligned}
$$

Bearing in mind the definition of $X$ and $Y$ and the relation between Poisson brackets and quantum mechanical commutation relations, this corresponds to the 
commutation relations

$$
\begin{aligned}
& {\left[\operatorname{Tr} A \frac{d g}{d \sigma} g^{-1}(\sigma), \operatorname{Tr} B \frac{d g}{d \sigma} g^{-1}\left(\sigma^{\prime}\right)\right]} \\
& \quad=\frac{4 \pi}{n} i \delta\left(\sigma-\sigma^{\prime}\right) \operatorname{Tr}[A, B] \frac{d g}{d \sigma} g^{-1}+\frac{4 \pi}{n} i \delta^{\prime}\left(\sigma-\sigma^{\prime}\right) \operatorname{Tr} A B .
\end{aligned}
$$

Now, let us compare this to the Kac-Moody algebra (21). We see that they coincide if $k=n$ and if $J_{-}$is identified with $\frac{n}{2 \pi} \frac{d g}{d \sigma} g^{-1}$.

Now, that conclusions can we draw? In the nonlinear sigma model and in Eq. (30), $n$ is an integer because of the multivaluedness of the Wess-Zumino coupling. In (21) $k$ is an integer because only then does the Kac-Moody algebra have well-behaved unitary representations. We see that single valuedness of $e^{i I}$, required in quantum mechanics for mathematical consistency, leads to a KacMoody algebra with properly normalized central charge.

Second, the theory at $\lambda^{2}=4 \pi / n$ really does have a vanishing $\beta$ function, because it is known [15] that the irreducible representation of the Kac-Moody algebra is conformally invariant (can be extended to the semi-direct product of the KacMoody algebra with the conformal algebra).

Third, and most important, it follows from Eq. (30) that the non-linear sigma model with $n=1$ and $\lambda^{2}=4 \pi$ is equivalent to the free field theory of $N$ massless Majorana fermions. For, with the identifications

$$
\begin{aligned}
& J_{-}^{i j}=i \psi_{-}^{i} \psi_{-}^{j}=\frac{1}{2 \pi}\left(\frac{d g}{d \sigma} g^{-1}\right)^{i j}, \\
& J_{+}^{i j}=i \psi_{+}^{i} \psi_{+}^{j}=\frac{1}{2 \pi}\left(g^{-1} \frac{d g}{d \tau}\right)^{i j},
\end{aligned}
$$

the currents of these theories obey the same algebra [Eqs. (20) and (30)], and, as has been mentioned, this algebra has an essentially unique irreducible representation. (The Hilbert spaces of the quantum theories in question furnish irreducible representations of the current algebras because no operators commute with all the currents. This has been proved [13] in the fermi case and also [16] in the bose case. ${ }^{6}$ ) The Kac-Moody representation is not quite unique, but the nonuniqueness just refers to superselection rules and boundary conditions in the quantum field theory.

Moreover, the Hamiltonian $H$ and the momentum operator $P$ of the free fermi theory coincide with those of the non-linear sigma model at the special values of

6 Our discussion of the non-linear sigma model is closely related to the discussion of the Kac-Moody representations in [16]. The phase space of our theory in the light cone frame is a complex manifold, the loop space $Z$ of $\mathrm{O}(N)$. (Actually, this is only half the phase space of the theory, since it omits leftmoving waves. The full phase space is $Z \times Z$.) The operator $F_{i j}$ that we have constructed represents the first Chern class of a holomorphic line bundle $E$ over $Z$. The Hilbert space of the theory is the space of holomorphic sections of $E$. This construction generalizes some classical theorems about representations of finite dimensional Lie groups to the Kac-Moody system. The novelty of our discussion of the non-linear sigma model is to show that the construction of Kac-Moody representations just mentioned can be realized by canonical quantization of a quantum field theory 
the couplings under discussion. This can be seen in various ways. Because the Hilbert spaces form irreducible representations of the current algebras, $H$ and $P$ are uniquely determined in each case by their commutation relations with the currents. These commutation relations are suitable; $H$ and $P$ generate translations in both the bose and fermi theories. A more explicit argument is the following. In the fermi theory one can show $H+P=$ const $\lim _{\varepsilon \rightarrow 0} \int d x J_{+}^{i j}(x+\varepsilon) J_{+}^{i j}(x)$, and a similar formula for $H-P$ in terms of $J_{-}$. (This is not a canonical equation. One must study the short distance behavior of the product of currents, and subtract an infinite $c$-number.) On the other hand, in the bose theory one can calculate canonically an equivalent formula

$$
H+P=\text { const } \int d x \operatorname{Tr}\left(g^{-1} \frac{d g}{d \tau}\right)^{2}
$$

(and similarly for $H-P$ ). In view of (31) these relations show that $H$ and $P$ of the bose theory equal those of the fermi theory.

By introducing several fermion flavors, it is possible to make a bose-fermi translation also for $n \neq 1$. Consider a theory with $N$ "colors" and $n$ "flavors" of Majorana fermions $\psi^{i a}, i=1 \ldots N$ and $a=1 \ldots n$. We can define currents $\tilde{J}_{ \pm}^{i j}=-i \sum_{a} \psi_{ \pm}^{i a} \psi_{ \pm}^{j a}, \quad \tilde{J}_{ \pm}^{a b}=-i \sum_{i} \psi_{ \pm}^{i a} \psi_{ \pm}^{i b}$. These currents generate $\mathrm{O}(N)_{L}$ $\times \mathrm{O}(N)_{R} \times \mathrm{O}(n)_{L} \times \mathrm{O}(n)_{R}$. The $\mathrm{O}(N)_{L} \times \mathrm{O}(N)_{R}$ current commutators have anomalies of strength $n$. The $\mathrm{O}(n)_{L} \times \mathrm{O}(n)_{R}$ current commutators have anomalies of strength $N$. The free field theory Hilbert space is an irreducible representation [17] of the combined current algebra.

An equivalent bose theory is a theory with two fields $g$ and $h ; g$ takes values in $\mathrm{O}(N)$ and $h$ in $\mathrm{O}(n)$. For $g$ we take a Wess-Zumino coupling $n$ and $\lambda^{2}=4 \pi / n$; for $h$ we take a Wess-Zumino coupling $N$ and $\lambda^{2}=4 \pi / N . g$ and $h$ are decoupled, corresponding to the fact that at the fermion level amplitudes with a product of $\mathrm{O}(N)_{L} \times \mathrm{O}(N)_{R} \times \mathrm{O}(n)_{L} \times \mathrm{O}(n)_{R}$ currents factorize [18] as a product of $\mathrm{O}(N)_{L} \times \mathrm{O}(N)_{R}$ amplitudes and $\mathrm{O}(n)_{L} \times \mathrm{O}(n)_{R}$ amplitudes. The Hamiltonian and momentum operators of $\psi^{i a}$ can be identified with the sum of those constructed from $g$ and $h$.

As we have discussed, Eq. (31) generalizes to the non-abelian case the equation $\bar{\psi} \gamma_{\mu} \psi=\frac{1}{\sqrt{\pi}} \varepsilon_{\mu \nu} \partial^{v} \phi$ of conventional bosonization. In conventional bosonization, there also are formulas $\bar{\psi} \psi=M \cos \sqrt{4 \pi} \phi, \bar{\psi} i \gamma_{5} \psi=M \sin \sqrt{4 \pi} \phi$ ( $M$ is a renormalization mass). What are the analogues of those formulas here?

Let $Q_{k}^{i}=-i \psi_{-}^{i} \psi_{+k}$. We would like to translate $Q_{k}^{i}$ into the bose language. The commutators of $Q^{i} k$ with the fermion currents are as follows:

$$
\begin{aligned}
& {\left[J_{i j}^{-}(x), Q_{l}^{k}(y)\right]=-i \delta(x-y)\left(\delta_{j k} Q_{l}^{i}(y)-\delta_{i k} Q_{l}^{j}(y)\right],} \\
& {\left[J_{i j}^{+}(x), Q_{l}^{k}(y)\right]=-i \delta(x-y)\left(\delta_{j l} Q_{i}^{k}(y)-\delta_{i l} Q_{j}^{k}(y)\right) .}
\end{aligned}
$$

We must therefore find in the bose theory operators obeying the algebra (32). One need not look far. The matrix elements $g_{j}^{i}(x)$ of the matrix $g$ are the required 
operators. Canonically,

$$
\begin{aligned}
& {\left[\frac{1}{2 \pi}\left(\frac{d g}{d \sigma} g^{-1}(x)\right)^{i j}, g_{l}^{k}(y)\right]=-i \delta(x-y)\left(\delta^{j k} g_{l}^{i}(y)-\delta^{i k} g_{l}^{j}(y)\right)} \\
& {\left[\frac{1}{2 \pi}\left(g^{-1} \frac{d g}{d \tau}(x)\right)^{i j}, g_{l}^{k}(y)\right]=-i \delta(x-y)\left(\delta^{j l} g_{i}^{k}(y)-\delta^{i l} g_{j}^{k}(y)\right)}
\end{aligned}
$$

The evaluation of (33) is simple for the following reason. From (30) we know that $\int d x \frac{d g}{d \sigma} g^{-1}$ and $\int d x g^{-1} \frac{d g}{d \tau}$ generate chiral $\mathrm{O}(N) \times \mathrm{O}(N)$. Therefore, (33) holds up to total derivatives that vanish after integrating over $x$. By virtue of locality and dimensional analysis $-g$ is dimensionless - there are no such possible terms. For the same reason, there can be no anomaly in (33) quantum mechanically.]

At least heuristically, it appears that $Q_{k}^{i}(x)$ and $g_{k}^{i}(x)$ are uniquely characterized (up to normalization) by the relations (32) and (33). For instance, in the free fermi theory one cannot find another operator that transforms like $Q_{k}^{i}(x)$, so we are led to conclude

$$
Q_{j}^{i}(x)=-i \psi_{-}^{i} \psi_{j+}(x)=M g_{j}^{i}(x),
$$

where (as in the conventional bosonization) $M$ is a mass that depends on the renormalization procedure for the bosonic operator. It should be noted that while $-i \psi_{-}^{i} \psi_{+}^{j}$ has canonical dimension one $-g_{j}^{i}$ is dimensionless classically. So Eq. (34) is possible only if $g_{j}^{i}$ has anomalous dimension one in the fixed point theory with $n=1$.

Equation (34) is a generalization of the conventional bosonization formulas, in which $\bar{\psi} \psi$ and $\bar{\psi} i \gamma_{5} \psi$ are identified with matrix elements of the $\mathrm{O}(2)$ matrix $\left(\begin{array}{rr}\cos \sqrt{4 \pi} \phi & \sin \sqrt{4 \pi} \phi \\ -\sin \sqrt{4 \pi} \phi & \cos \sqrt{4 \pi} \phi\end{array}\right)$. Of course, in the free bose field theory, it is easy to see that $\cos \sqrt{4 \pi} \phi$ and $\sin \sqrt{4 \pi} \phi$ do have anomalous dimension one.

Equation (34) can be tested in the following way. We have identified $i \psi_{i}^{-} \psi_{j}^{-}$ with $\frac{1}{2 \pi}\left(\frac{d g}{d x^{-}} g^{-1}\right)_{j}^{i}=\frac{1}{2 \pi} \sum_{k} \frac{d}{d x^{-}} g_{k}^{i}\left(g^{-1}\right)_{j}^{k}$. Since $g$ is orthogonal, $\left(g^{-1}\right)_{j}^{k}=g_{k}^{j}$. So $i \psi_{i}^{-} \psi_{j}^{-}=\frac{1}{2 \pi} \sum_{k} \frac{d g_{k}^{i}}{d x^{-}} g_{k}^{j}$. If we identify $g_{j}^{i}$ with $\frac{-\mathrm{i}}{\mathrm{M}} \psi_{-}^{i} \psi_{j+}$, we are led to require

$$
i \psi_{i}^{-} \psi_{j}^{-}(x)=-\frac{1}{2 \pi M^{2}} \sum_{k}\left(\frac{d}{d x^{-}}\left(\psi_{i}^{-} \psi_{k}^{+}(x)\right)\right)\left(\psi_{j}^{-} \psi_{k}^{+}(x)\right)
$$

At first sight Eq. (35) looks preposterous. It relates an operator quadratic in spinors to one quartic in spinors. However, to understand the right-hand side of (35), we must study the small $\Delta$ behavior of

$$
\frac{1}{\Delta^{2}} \int_{\substack{y^{0}-x^{0} \mid<\Delta / 2 \\ y^{1}-x^{1}}} d^{2} y \frac{\partial}{\partial y^{-}} T\left(\psi_{i}^{-} \psi_{k}^{+}(y) \psi_{j}^{-} \psi_{k}^{+}(x)\right)
$$


Since $\sum_{k=1}^{N} \frac{\partial}{\partial y^{-}} T\left(\psi_{k}^{+}(y) \psi_{k}^{+}(x)\right)=N \delta^{2}(x-y),(36)$ has a piece $-\frac{N}{\Delta^{2}} \psi_{i}^{-} \psi_{j}^{-}(x)$. This is the most singular part of (36), as $\Delta \rightarrow 0$. Equation (35) must be understood to mean that the operator on the left-hand side equals the most singular part of the operator on the right-hand side. Note that while $\Delta$ is cut-off dependent, the mass $M$ appearing in $-i \psi_{i}^{-} \psi_{j}^{+}(x)=M g_{j}^{i}(x)$ is also cutoff dependent [since $-i \psi_{i}^{-} \psi_{j}^{+}$has no anomalous dimension, but as already noted $g_{j}^{i}$ must have anomalous dimension one in the equivalent bose theory, if the relation $-i \psi_{i}^{-} \psi_{j}^{+}(x)=M g_{j}^{i}(x)$ holds]. Evidently, in view of (35) and (36), the product $M \Delta$ is cut-off independent. Equation (35) holds in the limit as $M \rightarrow \infty$ and $\Delta \rightarrow 0$ with $M \Delta$ fixed. While these manipulations are somewhat bizarre, the same bizarre manipulations are needed in conventional bosonization to show the consistency of the relations

$$
\bar{\psi} \gamma^{\mu} \psi=\frac{1}{\sqrt{\pi}} \varepsilon^{\mu v} \partial_{v} \phi, \quad \bar{\psi} \psi=M \cdot \cos \sqrt{4 \pi} \phi, \quad \bar{\psi} i \gamma_{5} \psi=M \sin \sqrt{4 \pi} \phi .
$$

This completes the dictionary of bosonization of fermi bilinears. Given the dictionary, it should be clear that the bosonization can be carried out also for arbitrary massive or interacting fermi theories. For instance, a fermion bare mass $m \bar{\psi} \psi=m i \sum_{k=1}^{N} \psi_{-}^{k} \psi_{+}^{k}$ can be included by adding to the Lagrangian a term proportional to $\sum_{i=1}^{N} g_{i}^{i}=\operatorname{Tr} g . \mathrm{A}(\bar{\psi} \psi)^{2}$ coupling becomes $(\operatorname{Tr} g)^{2}$. One can likewise study gauge theories in this way. For instance, in the fermion language one may choose to gauge an arbitrary anomaly free subgroup $H$ of chiral $\mathrm{O}(N) \times \mathrm{O}(N)$. The corresponding theory can be studied in the bose language by gauging the same subgroup $H$ of the symmetry group of the nonlinear sigma model. One will be limited to anomaly free groups $H$ - as one should be - because only for anomaly free groups does the Wess-Zumino term have a gauge invariant generalization [8].

Various applications of the present work can be imagined, but will not be explored here. The non-abelian bosonization may help in understanding $1+1$ dimensional field theories. It may be helpful in understanding the Callan-Rubakov effect, which is described by an effective $1+1$ dimensional $s$-wave field theory. And the conformally invariant theory with $\lambda^{2}=4 \pi / n$ may provide a starting point for constructing generalizations of the usual string theories.

\section{Appendix: Explicit Form of the Wess-Zumino Functional}

In this appendix we will work out an explicit formula for the Wess-Zumino functional in the simplest case of an SU(2) non-linear sigma model in two spacetime dimensions. We will use an index free notation, so antisymmetric tensors $\omega_{i j k}$ are denoted simply as $\omega$, and the curl of $\omega,\left(\partial_{i} \omega_{j k l} \pm\right.$ cyclic permutations $)$ is denoted $d \omega$. Differentials are considered to anticommute, so $d x d y=-d y d x$ and $(d x)^{2}=0$, if $x$ and $y$ are functions.

First let us write the Wess-Zumino functional in an abstract form. On the group manifold of any simple, non-abelian group $G$, there is a $G \times G$ invariant third 
rank tensor field $\omega$. $\omega$ obeys $d \omega=0$, and locally but not globally $\omega=d \lambda$ for some second rank tensor field $\lambda$. $\omega$ may be normalized so that its integral over any three sphere in $G$ is an integral multiple of $2 \pi$.

Let $B$ be a three-dimensional ball whose boundary, the two sphere $S$, is identified with space time. Given a mapping $g$ from $S$ into $G$, which has been extended to a mapping (also denoted $g$ ) from $B$ into $G$, the Wess-Zumino functional is defined as

$$
\Gamma=\int_{B} g^{*} \cdot \omega=\int_{B} g^{*} \cdot d \lambda=\int_{\partial B} g^{*} \cdot \lambda=\int_{S} g^{*} \cdot \lambda .
$$

Here $g^{*}$ is the "pull-back" of differential forms. In the third step of (37) $\partial B=S$ is the boundary of $B$; Stokes' theorem has been used. The last formula in (37) exhibits $\Gamma$ as the integral of an ordinary two-dimensional Lagrangian. In concrete terms, the meaning of this formula as follows. Let $\phi^{i}$ be a set of coordinates for the group manifold $G$. Let $\lambda_{i j}$ be the components of the anti-symmetric tensor $\lambda$. Then the mapping $g: S \rightarrow G$ can be described by means of functions $\phi^{i}$, and

$$
\Gamma=\int d^{2} x \varepsilon^{\mu v} \lambda_{i j}\left(\phi^{k}(x)\right) \partial_{\mu} \phi^{i} \partial_{\nu} \phi^{j}
$$

Equation (38) has "Dirac string" type singularities, because the defining equation of $\lambda, \omega=d \lambda$, can be solved only locally on the group manifold.

Equation (38) is $G \times G$ invariant, although not manifestly so. Under a $G \times G$ transformation $\lambda$ transforms as $\lambda_{i j} \rightarrow \lambda_{i j}+\frac{\partial \beta_{j}}{\partial \phi^{i}}-\frac{\partial \beta_{i}}{\partial \phi^{j}}$ for some $\beta_{i}\left(\phi^{k}\right)$. So

$$
\delta \Gamma=\int d^{2} x \varepsilon^{\mu v}\left(\partial_{i} \beta_{j}-\partial_{j} \beta_{i}\right) \partial_{\mu} \phi^{i} \partial_{\nu} \phi^{j}=2 \int d^{2} x \frac{\partial}{\partial x^{\mu}}\left(\varepsilon^{\mu v} \beta_{j} \partial_{\nu} \phi^{j}\right)
$$

and $\Gamma$ changes by a total divergence whose integral vanishes.

Now let us construct explicit formulas for the simplest non-abelian group $\mathrm{SU}(2)$. The $\mathrm{SU}(2)$ manifold is a three sphere; it can be described by polar angles $\psi$, $\theta, \phi$ with line element $d s^{2}=d \psi^{2}+\sin ^{2} \psi\left(d \theta^{2}+\sin ^{2} \theta d \phi^{2}\right)$. The only $\operatorname{SU}(2) \times \operatorname{SU}(2)$ invariant third rank antisymmetric tensor is the Levi-Civita tensor or volume form, so

$$
\omega=\frac{1}{\pi} \sin ^{2} \psi \sin \theta d \psi d \theta d \phi
$$

Note the normalization of (40). Since the volume of the SU(2) manifold is $2 \pi^{2},(40)$ is chosen so that the integral of $\omega$ over the whole manifold is $2 \pi$.

The equation $\omega=d \lambda$ can be solved in many ways, for instance

$$
\lambda=\frac{1}{\pi} \phi \sin ^{2} \psi \sin \theta d \psi d \theta
$$

[Recall $(d \psi)^{2}=(d \theta)^{2}=0$.] So given a mapping of space-time into $\mathrm{SU}(2)$, the properly normalized Wess-Zumino interaction is

$$
\Gamma=\frac{1}{\pi} \int d^{2} x \phi(x) \sin ^{2} \psi(x) \sin \theta(x) \varepsilon^{\mu v} \partial_{\mu} \psi(x) \partial_{v} \theta(x) .
$$


[In this parametrization, the Dirac-string type singularities occur at $\theta(x)=0$ or $\pi$. For at $\theta=0$ or $\pi$, everything should be independent of $\phi$. This is not true in Eq. (42). In the case of the group $\mathrm{SU}(2)$, it is possible to choose another parametrization that is singular only at a single point on the group manifold.]

Formulas similar to (42) can be constructed for other groups and also for the Wess-Zumino interaction in four dimensions. These formulas are not very enlightening, however.

Acknowledgements. I wish to thank A. Feingold and I. Frenkel for discussions about Kac-Moody algebras, and D. J. Gross for suggesting a test of Eq. (34). I also wish to acknowledge discussions of bosonization and related matters with S. Coleman and D. Olive.

\section{References}

1. Coleman, S.: Quantum sine-Gordon equation as the massive Thirring model. Phys. Rev. D11, 2088 (1975)

2. Mandelstam, S. : Soliton operators for the quantized sine-Gordon equation. Phys. Rev. D 11, 3026 (1975)

3. Baluni, V.: The Bose form of two-dimensional quantum chromodynamics. Phys. Lett. 90 B, 407 (1980)

Steinhardt, P.J.: Baryons and baryonium in QCD $_{2}$. Nucl. Phys. B 176, 100 (1980)

Amati, D., Rabinovici, E. : On chiral realizations of confining theories. Phys. Lett. 101 B, 407 (1981)

4. Wess, J., Zumino, B.: Consequences of anomalous word identities. Phys. Lett. 37 B, 95 (1971)

5. D'Adda, A., Davis, A.C., DiVecchia, P.: Effective actions in non-abelian theories. Phys. Lett. 121 B, 335 (1983)

6. Polyakov, A.M., Wiegmann, P.B.: Landau Institute preprint (1983)

7. Alvarez, O.: Berkeley preprint (1983)

8. Witten, E.: Global aspects of current algebra. Nucl. Phys. B (to appear)

9. Novikov, S.P.: Landau Institute preprint (1982)

10. Polyakov, A.M.: Interaction of Goldstone particles in two dimensions. Applications to ferromagnets and massive Yang-Mills fields. Phys. Lett. 59 B, 79 (1975)

Belavin, A.A., Polyakov, A.M.: Metastable states of two-dimensional isotropic ferromagnets. JETP Lett. 22, 245 (1975)

11. Nappi, C.R.: Some properties of an analog of the chiral model. Phys. Rev. D 21, 418 (1980)

12. Goto, T., Imamura, I.: Note on the non-perturbation-approach to quantum field theory. Prog. Theor. Phys. 14, 396 (1955)

Schwinger, J.: Field-theory commutators. Phys. Rev. Lett. 3, 296 (1959)

Jackiw, R.: In: Lectures on current algebra and its applications, Treiman S.B., et al. (eds.): Princeton, NJ: Princeton University Press 1972

Coleman, S., Gross, D., Jackiw, R.: Fermion avatars of the Sugawara model. Phys. Rev. 180, 1359 (1969)

13. Kac, V.G.: J. Funct. Anal. Appl. 8, 68 (1974)

Lepowsky, J., Wilson, R.L.: Construction of the affine Lie algebra. $A_{1}(1)$. Commun. Math. Phys. 62, 43 (1978)

Frenkel, I.B.: Spinor representations of affine Lie algebras. Proc. Natl. Acad. Sci. USA 77, 6303 (1980); J. Funct. Anal. 44, 259 (1981)

Feingold, A.J., Frenkel, I.B.: IAS preprint (1983)

14. Belavin, A.M., Polyakov, A.M., Schwar, A.S., Tyupkin, Yu.S.: Pseudoparticle solutions of the Yang-Mills equations. Phys. Lett. 59 B, 85 (1975)

't Hooft, G.: Symmetry breaking through Bell-Jackiw anomalies. Phys. Rev. Lett. 37, 8 (1976); Computation of the quantum effects due to a four-dimensional pseudoparticle. Phys. Rev. D 14, 3432 (1976)

Callan, C.G., Jr., Dashen, R., Gross, D.J.: The structure of the gauge theory vacuum. Phys. Lett. 63B, 334 (1976)

Jackiw, R., Rebbi, C.: Vacuum periodicity in a Yang-Mills quantum theory. Phys. Rev. Lett. 37, 172 (1976) 
15. Segal, G.: Unitary representations of some infinite-dimensional groups. Commun. Math. Phys. 80, 301 (1981)

Frenkel, I., Kac, V.G.: Basic representations. Invent Math. 62, 23 (1980)

16. Kac, V.G., Peterson, D.H.: Spin and wedge representations of infinite-dimensional Lie algebras and groups. Proc. Natl. Acad. Sci. USA 78, 3308 (1981)

17. Frenkel, I.: Private communication

18. Frishman, Y.: Quark trapping in a model field theory. Mexico City 1973. Berlin, Heidelberg, New York: Springer 1975

19. Deser, S., Jackiw, R., Templeton, S.: Three-dimensional massive gauge theories. Phys. Rev. Lett. 48, 975 (1982); Topologically massive gauge theories. Ann. Phys. (NY) 140, 372 (1982)

Communicated by A. Jaffe

Received September 29, 1983 